\title{
Indicadores epidemiológicos e distribuição espaço-temporal da tuberculose em município endêmico
}

RESUMO | Objetivo: descrever indicadores epidemiológicos da tuberculose e analisar a distribuição espaço-temporal. Métodos: Estudo ecológico, realizado com dados do Sistema de Informação de Agravos de Notificação. Variáveis: taxa de incidência; mortalidade; forma clínica; coinfecção tuberculose/HIV; cura e abandono. Para análise utilizou-se regressão linear simples. As taxas brutas de incidência foram suavizadas pelo Método Bayesiano Empírico Global e Local. Resultados: Foram estudados 5.529 casos e 319 óbitos. A forma pulmonar alcançou 96,2/100 mil habitantes, a coinfecção tuberculose/HIV 10,6/100 mil habitantes, o menor percentual de cura foi 69,0\% e, o maior percentual de abandono, 10,9\%. O Bayes Global indicou padrão irregular e heterogêneo da doença e, o Bayes local revelou maior concentração de casos em algumas regiões. Conclusão: A tuberculose se distribui de forma não aleatória e seu controle permanece um desafio.

Palavras-chaves: Tuberculose; Sistemas de Informação Geográfica; Análise Espacial; Epidemiologia; Saúde Pública.

ABSTRACT | Objective: To describe the epidemiological indicators of tuberculosis and to analyze space-time distribution. Methods: Ecological study, carried out with data from the Grievances Notification Information System. Variables: incidence rate; mortality; clinical form; tuberculosis/HIV co-infection; cure and abandonment. For analysis we used the simple linear regression, Gross incidence rates were softened by the Bayesian Empirical Global and Local Method. Results: A total of 5,529 cases and 319 deaths were studied. The pulmonary form reached 96.2/100 thousand inhabitants, the co-infection tuberculosis/HIV, 10.6/100 thousand inhabitants, the lowest percentage of cure was $69.0 \%$ and, the highest percentage of abandonment, $10.9 \%$. Global Bayes indicated an irregular and heterogeneous pattern of the disease and the local Bayes revealed a higher concentration of cases in some regions. Conclusion: Tuberculosis is distributed non-randomly and its control remains a challenge.

Keywords: Tuberculosis; Geographic Information Systems; Spatial Analysis; Epidemiology; Public Health.

RESUMEN | Objetivo: describir los indicadores epidemiológicos de la tuberculosis y analizar distribución espacio-temporal. Métodos: Estudio ecológico, realizado con datos del Sistema de Información de Enfermedades de Notificación. Variables: tasa de incidencia; mortalidad; forma clínica; coinfección por tuberculosis $\mathrm{NIH}$; curación y abandono. Para el análisis, se utilizó regresión lineal simple. Las tasas brutas de incidencia se suavizaron mediante el Método Bayesiano Empírico Local y Global. Resultados: se estudiaron 5.529 casos y 319 defunciones. La forma pulmonar alcanzó 96,2 / 100 mil habitantes, coinfección tuberculosis / VIH 10,6 / 100 mil habitantes, el menor porcentaje de curación fue 69,0\% y el mayor porcentaje de abandono 10,9\%. Bayes Global indicó un patrón irregular y heterogéneo de la enfermedad, y Bayes local reveló una mayor concentración de casos en algunas regiones. manera espacial, indican heterogeneidad en la Conclusión: La tuberculosis se distribuye de forma no aleatoria y su control sigue siendo un desafío.

Palabras claves: Tuberculosis; Sistemas de Información Geográfica; Análisis espacial; Epidemiología; Salud pública.

\section{Ingrid Bentes Lima}

Acadêmica de Enfermagem da Universidade do Estado do Pará (UEPA). Belém, PA. ORCID: 0000-0001-7717-8219

\section{Laura Maria Vidal Nogueira}

Enfermeira, Pós-doutora em Enfermagem. Professora adjunto do departamento de enfermagem comunitária da Universidade do Estado do Pará (UEPA). Coordenadora de atividades do programa de Pós-graduação - Mestrado acadêmico associado UEPA/UFAM. Belém (PA), Brasil.

ORCID: 0000-0003-0065-4509

\section{Claudia Benedita dos Santos}

Matemática. Pós-doutora. Professora Associada da Universidade de Ribeirão Preto da Universidade de São Paulo (EERP/USP). Ribeirão Preto (SP), Brasil.

ORCID: 0000-0001-7241-7508

\section{Ivaneide Leal Ataide Rodrigues}

Enfermeira. Pós-doutora em Enfermagem. Professora adjunto do departamento de enfermagem comunitária da Universidade do Estado do Pará (UEPA). Docente do quadro permanente do programa de Pós-graduação - Mestrado acadêmico associado UEPAVUFAM. Belém (PA), Brasil.

ORCID: 0000-0001-9968-9546

\section{Lidiane de Nazaré Mota Trindade}

Enfermeira. Mestre em Enfermagem. Professora substituta na Universidade do Estado do Pará (UEPA). Belém (PA), Brasil.

ORCID: 0000-0003-2202-8138

Recebido em: 18/05/2021

Aprovado em: 07/06/2021

\section{Suzana Rosa André}

Enfermeira. Doutoranda em Enfermagem pela Universidade Federal do Rio de Janeiro, Escola de Enfermagem Anna Nery. Rio de Janeiro (RJ), Brasil.

ORCID: 0000-0002-4219-9279

\section{INTRODUÇ̃̃̃O}

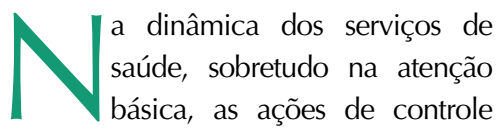
da tuberculose (TB) têm sido fortemente absorvidas pela enfermagem, desde o acolhimento do sintomático respiratório até a alta medicamentosa da pessoa que recebeu o tratamento específico. O Ministério da Saúde considera ser atribuição do enfermeiro: identificar sintomáticos respiratórios, reali- 
zar consulta de enfermagem, orientar quanto à coleta de escarro, prescrever o esquema básico, notificar casos de TB, entre outras. ${ }^{1}$

O perfil epidemiológico da doença em países classificados com baixa e média renda que exibem elevadas taxas de adoecimento e mortalidade, preocupam autoridades sanitárias, que sistematicamente vem apresentando planos e estratégias para conter sua disseminação. Segundo a Organização Mundial da Saúde (OMS), as maiores taxas de mortalidade por doenças infecciosas no mundo são por TB, situando-se acima do HIV. Em 2018, ocorreram 10 milhões de casos da doença no mundo. ${ }^{2} \mathrm{O}$ Brasil por ser um país de área endêmica da doença encontra-se entre os 30 países com maior número de casos. ${ }^{2}$

Em 2014, a OMS traçou diretrizes internacionais de controle da TB, conhecidas como Estratégia End TB, em consonância com os Objetivos de Desenvolvimento do Milênio (ODM), sucedidos pelos Objetivos de Desenvolvimento Sustentável (ODS) ${ }^{2,3}$, que tem como meta reduzir o número de óbitos em 95\% e a taxa de incidência em $90 \%$ até $2035 .{ }^{2}$, além de eliminar a doença como epidemia até 2030 . $^{3}$

Em 2019 foram notificados, no Brasil, 73.684 casos novos, com coeficiente de incidência de 35,0/100 mil habitantes e coeficiente de mortalidade de 2,2/100 mil habitantes. ${ }^{4}$ No mesmo ano, o Pará notificou 4.459 casos novos da doença, sendo o Estado com maior número de casos na região Norte. Já no município de Belém foram 1.354 casos e coeficiente de incidência de 90,7/100 mil habitantes, sendo a segunda capital com maior índice de mortalidade no país, 6,0/100 mil habitantes. ${ }^{4}$

A análise dos indicadores epidemiológicos, em conjunto com os recursos do Sistema de Informações Geográficas (SIG), pode ofertar dados para uma avaliação mais rigorosa e consequentemente um planejamento mais eficaz que leve a melhor controle da doença. As técnicas de análise espacial possibilitam representar, por meio de mapas, regiões geográficas com risco elevado de desenvolver doenças e permitem buscar associações da TB com fatores demográficos, epidemiológicos e ambientais. ${ }^{5}$ Nesse sentido, o presente estudo tem como objetivos descrever os indicadores epidemiológicos da TB e analisar sua distribuição espaço-temporal.

\section{MÉTODOS}

Estudo epidemiológico, ecológico desenvolvido com dados de TB, notificados na rede de serviços do município de Belém, no período de 2013 a 2016. Belém é capital prioritária para o controle da doença, com população estimada para 2019 de 1.492 .745 pessoas e densidade demográfica de 1.315,26 habitantes por quilômetro quadrado. ${ }^{6} \mathrm{O}$ presente artigo é resultado de projeto multicêntrico que investiga doenças negligenciadas em distintos municípios do Brasil. É derivado do estudo que explorou a tuberculose associada às condições de vida em Belém. ${ }^{7}$

Os dados são provenientes do Sistema de Informação de Agravos de Notificação (SINAN) e foram disponibilizados pela Secretaria Municipal de Saúde de Belém (SESMA). Os indicadores epidemiológicos estudados foram: taxa de incidência de TB todas as formas; taxa de incidência de TB extrapulmonar; taxa de incidência de TB pulmonar bacilífera; taxa de mortalidade por TB; proporção de casos de TB curados; proporção de casos de TB que abandonaram o tratamento e coeficiente de TB/HIV. E as variáveis sociodemográficas: ano de notificação; idade; sexo e escolaridade.

Como critérios de inclusão foram definidos todos os casos de TB notificados nas Unidades de Saúde do município de Belém no período de janeiro de 2013 a dezembro de 2016. De posse do banco dados foi feita crítica minuciosa não encontrando inconsistências nas variáveis do estudo, consequentemente não houve exclusão de casos. Nessa ocasião, as variáveis foram organizadas e agrupadas por meio do Microsoft Office Excelß 2010.

O processo de análise desenvolveu-se em quatro etapas: na primeira foi feita a descrição dos indicadores epidemioló- gicos segundo as variáveis: sexo; idade e escolaridade.

$\mathrm{Na}$ segunda etapa realizou-se o cálculo das taxas de incidência e percentual dos indicadores epidemiológicos dos casos novos de TB notificados ao SINAN no período de 2013 a 2016, utilizando o dado populacional disponibilizado pelo IBGE, oriundo do censo 2010. Os cálculos foram realizados por 100.000 habitantes. Para identificação da tendência dos indicadores estudados foi realizada análise de regressão linear simples, sendo que o ajuste da função de regressão foi avaliado pelo coeficiente de determinação (R2) considerando nível de significância de 5\%, utilizando o programa estatístico Bioestat 5.4.

$\mathrm{Na}$ terceira etapa construiu-se um banco de dados geográficos (BDGEO) com os casos notificados. Para tanto, realizou-se a formatação e correção ortográfica da planilha com os endereços de residência. Os arquivos vetoriais das bases cartográficas digitais, por malhas do setor censitário do município de Belém foram obtidos no site do IBGE.

Em seguida, os endereços foram geocodificados na projeção Universal Transversa de Mercator (UTM), zona 22, onde se encontra o município de Belém, utilizando o site de geocodificação em lotes que usa a base de dados do Google Earth $\AA$ denominado "doogal.co.uk" (https://www.doogal. co.uk/BatchGeocoding.php). As análises geográficas foram realizadas por meio do software TerraView 4.2.2 do Instituto Nacional de Pesquisas Espaciais - INPE.

$\mathrm{Na}$ quarta etapa, foi aplicado o modelo Bayesiano empírico global e local considerando que os cálculos de taxas brutas de pequenas áreas geográficas são poucos indicados para a realização de análise espacial, pois podem sofrer influência da flutuação de cálculos em função de número reduzido de casos.

Os mapas temáticos foram construídos pelo software livre QGis versão 3.14, disponibilizado pela Open Source Geospacial Fundation (OSGEO).

Este estudo obedeceu ao estabelecido na Resolução n 466/12 do Conselho 
Nacional de Saúde. Iniciou-se após aprovação no Comitê de Ética em Pesquisa do Curso de Graduação em Enfermagem, em 15 de fevereiro de 2019, sob o parecer 3.148.828 e assinatura do Termo de Autorização de Acesso ao Banco de Dados-TAABD pelo técnico responsável por disponibilizar os dados na Secretaria de Saúde do Município de Belém.

\section{RESULTADOS}

No período de 2013 a 2016 foram registrados 5.529 casos novos de TB com tendência decrescente na incidência de 2,9 casos/100 mil hab. a cada ano. Quanto aos óbitos, foram 319, com redução até o ano 2015 e discreta elevação em 2016 (Tabela 1).

O perfil dos casos estudados revelou predomínio do sexo masculino, com $3.427(62 \%)$ casos e faixa etária de 15 a 29 anos, com 1.1815 (32,8\%) registros, seguido de 30 a 44 anos, com
1.650 (29,8\%). Em relação à escolaridade, 1.728 (30,8\%) apresentavam ensino fundamental incompleto, 1.646 (29,8\%) nível médio incompleto ou completo, 357 (6,5\%) nível superior incompleto ou completo, $339(6,1 \%)$ fundamental completo, $1.282(23,2 \%)$ apresentavam escolaridade ignorada e $121(2,2 \%)$ não a tinham.

Em relação a forma clínica, a mais prevalente foi a TB pulmonar (4.616 casos/83,5\%), com destaque para 2016 que alcançou $84,5 \%$ dos casos, seguida da TB extrapulmonar (733 casos/13,2\%) e TB pulmonar + extrapulmonar (180 casos/3,2\%). Nota-se que no período, as taxas da forma extrapulmonar reduziram e as da forma pulmonar elevaram-se (Tabela 2).

No que diz respeito aos desfechos de tratamento, 4.150 (75\%) casos evoluíram para cura, com redução de 3,0 casos/100 hab entre os anos estudados. Os registros de abandono de tratamento representaram $10,3 \%$ (571) dos casos notificados no período, sendo a menor proporção 9,7\% (134) em 2015 (Tabela 2).

De acordo com a tabela 3, os casos de coinfecção TB/HIV mostraram-se instáveis ao longo dos anos, crescentes de 2013 a 2015 e decrescentes de 2015 a 2016. A coinfecção foi detectada em 588 casos, com destaque para o ano de 2015 que apresentou ocorrência de 11,6 casos/100 hab., portanto, superior aos demais anos.

$\mathrm{Na}$ figura 1, observa-se o comportamento espacial da TB no munícipio de Belém a partir da aplicação do método Bayesiano Global, revelando um padrão heterogêneo e irregular de risco para o adoecimento. As taxas suavizadas oscilaram de 1.25 a 6.93 indicando variação decrescente. Os bairros mais periféricos como Jurunas, Guamá, Telégrafo, Sacramenta, Barreiro, Miramar, Cabanagem e Chapéu Virado apresentaram os maiores índices, com valores entre 3.95 e 6.93, os demais bairros, em sua maioria, apresentaram taxa entre 2.80 e 3.94. Os bairros

\section{Tabela 1. Proporção de casos novos e óbitos por TB no período de 2013 a 2016, no município de Belém. Belém, PA, Brasil, 2017.}

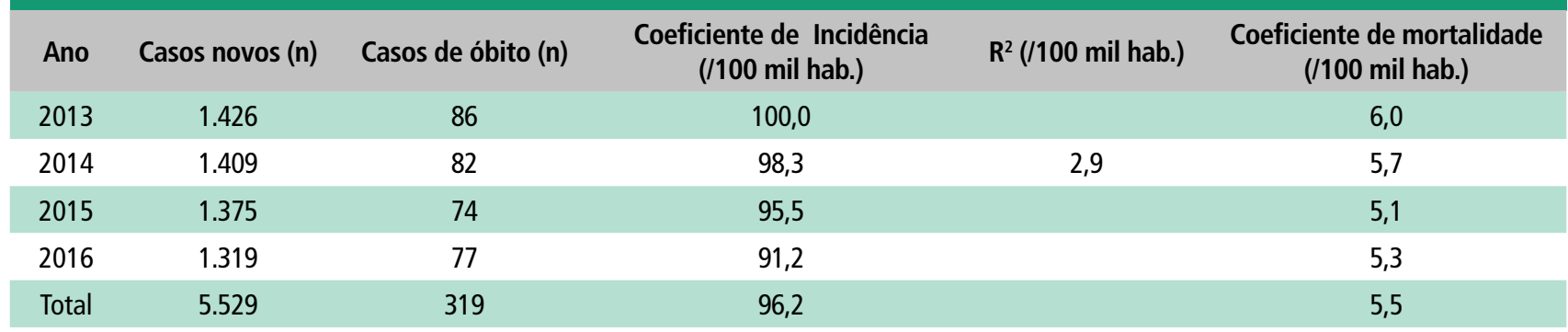

Fonte: SINAN/SESMA.

Tabela 2. Proporção de casos novos de TB segundo forma clínica, casos curados e de abandono de tratamento no período de 2013 a 2016, no município de Belém ( $\mathrm{n=5.529).} \mathrm{Belém,} \mathrm{PA,} \mathrm{Brasil,} 2017$.

\begin{tabular}{|c|c|c|c|c|c|c|c|c|c|c|c|}
\hline Ano & \multicolumn{2}{|c|}{2013} & \multicolumn{2}{|c|}{2014} & \multicolumn{2}{|c|}{2015} & \multicolumn{2}{|c|}{2016} & \multicolumn{3}{|c|}{ Total } \\
\hline \multicolumn{12}{|l|}{ Forma Clínica } \\
\hline Extra pulmonar & 189 & 13,3 & 194 & 13,8 & 184 & 13,4 & 166 & 12,6 & 733 & 13,2 & \\
\hline Pulmonar & 1.197 & 83,9 & 1167 & 82,8 & 1138 & 82,8 & 1.114 & 84,5 & 4616 & 83,5 & \\
\hline Cura & 1.107 & 77,6 & 1.112 & 78,9 & 1.025 & 74,5 & 910 & 69,0 & 4,150 & 75 & $-3,0$ \\
\hline Abandono & 155 & 10,9 & 140 & 9,9 & 134 & 9,7 & 142 & 10,8 & 571 & 10,3 & \\
\hline
\end{tabular}

Fonte: SINAN/SESMA. 
mais centrais apresentaram menores proporções de casos e de aglomerados anormais, tais como: Batista Campos, Nazaré e Reduto.

A distribuição de taxas suavizadas pelo método Bayesiano Local ilustrada na figura 2 indica variação de 0.00 a 20.0 nos índices. $\mathrm{O}$ índice local possibilitou outra configuração no mapa diferente da figu- ra 1, pois a maior parte das localidades apresentou tons mais claros, significando índices menores. Os bairros Guamá, Cremação, Condor, Jurunas e Miramar apresentaram os índices mais elevados, variando de 10.01 a 20.00. A análise Bayesiana local revelou maior concentração de casos em algumas regiões, configurando distribuição espacial não aleatória.
Tabela 3. Proporção de casos de coinfecção TB/HIV período de 2013 a 2016, no município de Belém. Belém, PA, Brasil, 2020.

\begin{tabular}{cccc} 
Ano & No $^{\circ}$ de casos & TB/HIV & Ocorrência TB/HIV \\
2013 & 1.426 & 144 & 10,1 \\
\hline 2014 & 1.409 & 154 & 10,9 \\
\hline 2015 & 1.375 & 160 & 11,6 \\
\hline 2016 & 1.319 & 130 & 9,9 \\
\hline Total & 5.529 & 588 & 10,6 \\
\hline
\end{tabular}

Fonte: SINAN/SESMA

\section{Figura 1. Mapa temático do Índice de Bayes Global de casos de tuberculose no} município de Belém, no período de 2013 a 2016. Belém, PA, Brasil, 2020.

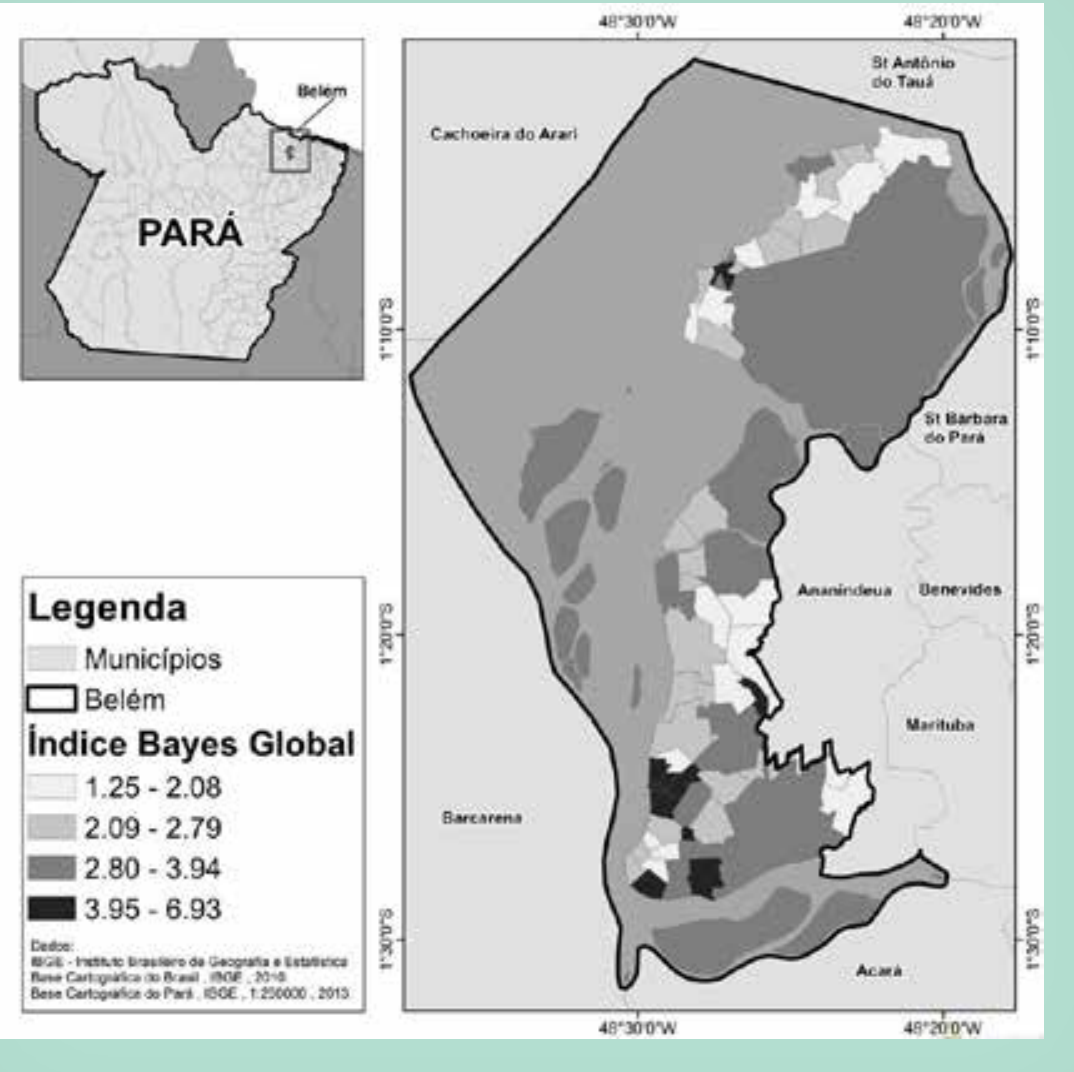

Fonte: SINAN/SESMA.

\section{DISCUSSÃO}

Os resultados do estudo indicam decréscimo anual no número de casos de TB no município de Belém e baixo desempenho do programa, visualizado nas proporções de cura e abandono ao tratamento. Os casos estão distribuídos aleatoriamente no espaço geográfico.

O perfil socioeconômico mostrou predominância do sexo masculino, também identificada em outros estudos, o que indica maior vulnerabilidade à doença, atribuído a dificuldade de acesso aos serviços de saúde, maior exposição aos fatores de risco e o autocuidado prejudicado. ${ }^{8,9}$

Identificou-se maior acometimento em adultos jovens, economicamente ativos, podendo incidir na renda familiar. Tal achado corrobora com estudo ${ }^{10}$ que identificou, dentre os fatores de risco para o adoecimento, as atividades laborais de constante contato com o público, bem como cargas horárias extensas. A TB permanece com maior incidência em grupos com baixo nível de escolaridade, evidenciado também em estudos que identificaram associação entre o grau de escolaridade e a renda familiar, bem como às condições de moradia, acesso a informações e aos serviços de saúde. ${ }^{11,12}$

A incidência da TB no município de Belém mostrou redução que pode ser explicada pela detecção precoce de casos, reduzindo o contágio, pela elevação de ações preventivas, ou ainda pela subnotificação nos registros. ${ }^{13}$ Entretanto, outras análises demonstraram elevação no coeficiente de incidência nos anos subsequentes do estudo: 2017, 2018 e 2019. ${ }^{4}$

Os óbitos reduziram de 2013 até 2015 , sendo que no último ano houve elevação no coeficiente de mortalidade. Estudo ${ }^{14}$ similar realizado no estado do Pará, no período 2006-2010 identificou 258 óbitos por TB, e menor incidência em comparação ao presente estudo. Outros achados, nas demais regiões do país e do mundo também demonstraram essa redução. ${ }^{15}$

Dentre os casos, a forma pulmonar alcançou entre $80 \%$ e $90 \%$, semelhante 
Figura 2. Estimador de Bayes Local dos casos de tuberculose no município de Belém, no período de 2013 a 2016. Belém, PA, Brasil, 2020.

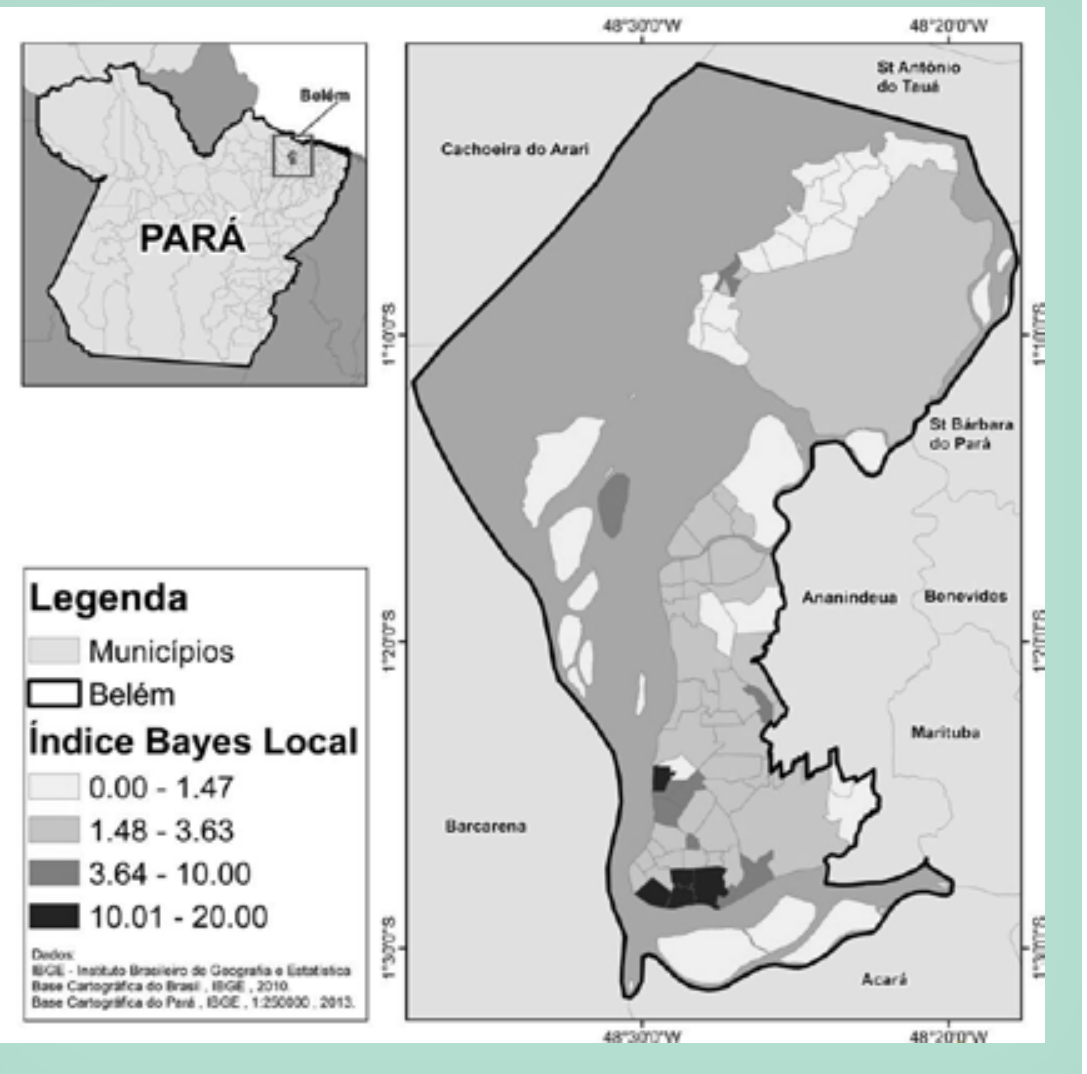

Fonte: SINAN/SESMA.

a outros estudos. ${ }^{9,16}$ Não obstante, a forma extrapulmonar é uma preocupação, sobretudo para os gestores públicos, pela maior complexidade para esclarecimento diagnóstico, tendo em vista que sua manifestação poderá ocorrer em diversos órgãos, cujos exames nem sempre estão disponíveis para pronto acesso pela população. ${ }^{16,17}$

Em relação ao resultado do tratamento, a cura apresentou redução nos últimos anos, ficando abaixo de $85 \%$, parâmetro preconizado pelo Ministério da Saúde. ${ }^{4} \mathrm{O}$ desfecho do tratamento acompanha os fatores socioeconômicos e comportamentais dos acometidos, embora os homens apresentem maior chance de cura, procuram menos o serviço de saúde. A baixa escolaridade também está associada à dificuldade de cura, destacando que o nível de escolarização reflete na economia familiar. ${ }^{18}$
Dentre os potencializadores para o atual panorama epidemiológico da TB, identifica-se a coinfecção TB/HIV cujos dados mostraram-se elevados com discreto aumento no último ano. Estudos atribuem esse padrão ao aumento de pessoas vivendo com HIV/aids (PVHA). ${ }^{19}$, 20 Para detecção da coinfecção, faz-se necessário testar todos os casos diagnosticados com TB, uma meta prejudicada na avaliação do programa em Belém, que, em 2019 obteve o quarto pior desempenho no contexto brasileiro, testando somente $62,0 \%$ dos casos, enquanto o país testou $76,2 \%$ dos casos diagnosticados. ${ }^{4}$

Os dados identificados denotam fragilidade na execução das ações de controle da TB, e, ao serem estudados de forma espacializada, indicam heterogeneidade na distribuição da doença. A maioria dos bairros com alta taxa de incidência são também os mais populosos e apresentam menor renda, ratificando resultados obtidos em estudos anteriores. 7,21 Trata-se de um cenário recorrente na avaliação do comportamento da doença, em que os maiores índices de adoecimento estão em cidades mais populosas, acometendo principalmente pessoas com baixa escolaridade, mais excluídas socialmente e pertencentes a famílias numerosas. ${ }^{8,21,22}$

Estudos explorando a temática encontraram essa dinamicidade e heterogeneidade da doença no espaço. ${ }^{14,21,23,24}$ Fortalecendo a explicação da espacialização heterogênea, pesquisa que analisou a associação da TB com Índice Adaptado de Condições de Vida identificou correlação positiva da doença com estratos populacionais em situação de exclusão social. ${ }^{7}$ Em adição, fatores sociodemográficos potencializam essa distribuição, remetendo a necessidade de valorização do acesso aos serviços de saúde para detecção precoce de casos e investigações mais precisas focadas na interrupção da transmissibilidade. ${ }^{21,22,23}$

A TB é uma doença transmitida por via aérea, portanto, sua ocorrência apresenta associação com a proximidade de áreas vizinhas com elevados índices da doença. ${ }^{24}$ A utilização do método Bayesiano permitiu reduzir a variabilidade dos dados de populações sob risco de transmissão e possibilitou a confecção de mapas mais informativos.

São necessários outros estudos para identificar associações dos indicadores epidemiológicos da TB com fatores socioespaciais, a fim de direcionar intervenções em áreas de alto risco e as práticas em saúde, sobretudo pela enfermagem, protagonista no desenvolvimento das ações de controle.

Esse estudo foi limitado por utilizar fonte de dados secundários, que podem apresentar deficiências de informações, seja pela subnotificação dos dados ou preenchimento inadequado, o que pode ter ocasionado possíveis perdas de geocodificação. 
A TB se comportou de forma aleatória entre os bairros, em sua maioria, com altas taxas bayesianas em territórios populosos e de baixa renda. Este cenário, que favorece a perpetuação da doença remete a necessidade de ações intersetoriais que contemplem programas/projetos que ofereçam moradias adequadas, acesso a renda, a educação e aos serviços de saúde de qualidade. Portanto, em que pese o protagonismo da enfermagem no enfren- tamento da TB, o alcance de impacto nos indicadores epidemiológicos e operacionais depende de ação conjugada entre governo e sociedade civil.

Os mapas temáticos e cálculos estáticos evidenciaram aspectos relevantes para o planejamento de ações e controle da TB. Essas análises indicam que o controle da doença proposto pelo PNCT ainda continua sendo um desafio para vigilância epidemiológica e para a assistência em saúde. Assim sendo, no plano de enfrentamento da TB, é essencial contemplar os vários aspectos res- ponsáveis pelo desencadeamento e manutenção da doença no meio social, de forma articulada com a ação dos profissionais de saúde, essencialmente da enfermagem.

A busca pelo sintomático respiratório, o diagnóstico e tratamento dos casos e o monitoramento dos indicadores, que são inerentes a rotina nos serviços de saúde devem estar conjugados com o enfrentamento das mazelas sociais e as necessidades individuais decorrentes da exclusão social, presentes em comunidades onde a doença é mais incidente.

\section{Referências}

1. Brasil. Ministério da Saúde. Secretaria de Vigilância em Saúde. Departamento de Vigilância Epidemiológica. Tratamento diretamente observado (TDO) da tuberculose na atenção básica: protocolo de enfermagem. Brasília: Ministério da Saúde, 2011. 2. World Health Organization-WHO. The End TB Strategy 2015 [Internet]. [cited 2020 aug 06].Avalaible from: https://www.who.int/tb/End_TB_brochure.pdf.

3. United Nations. Sustainable development goals: 17 goals to transform our world. [Internet]. 2015 [citado 2020 ago 06]. Disponível em: http://www.un.org/sustainabledevelopment/sustainable-development-goals.

4. BRASIL. Ministério da Saúde. Secretaria de Vigilância em Saúde. Número Especial | Mar. 2020 [Internet]. Brasília: Ministério da Saúde. 2020.

5. Beiranvand R, Karimi A, Delpisheh A, Sayehmiri K, Soleimani S, Ghalavandi S. Correlation Assessment of Climate and Geographic Distribution of Tuberculosis Using Geographical Information System (GIS). Iran J Public Health [Internet]. 2016 [cited 2020 nov 23]; 45 (1): 86-93. Avalaible from: https://www.ncbi.nlm.nih.gov/pmc/articles/PMC4822399/.

6. Instituto Brasileiro de Geografia e Estatística- IBGE. Cidades: Belém [Internet]. 2019 [citado 2020 jun 10]. Disponível em: https://www.ibge.gov.br/cidades-e-estados/pa/belem.html.

7. André SR, Nogueira LMV, Rodrigues ILA, Cunha TND, Palha PF, Santos CBD. Tuberculosis associated with the living conditions in an endemic municipality in the North of Brazil. Rev Lat Am Enfermagem [Internet]. 2020 [cited 2020 dec 12]; 28:e3343. Avalaible from: https://doi.org/10.1590/1518-8345.3223.3343.

8. Bergonzoli G, Castellanos LG, Rodríguez R, Garcia LM. Determinants of tuberculosis in countries of Latin America and the Caribbean. Rev Panam Salud Publica [Internet]. 2016 [cited 2020 oct 17]; 39 (2): 101-105. Avalaible from: HYPERLINK "https://www.scielosp.org/pdf/rpsp/2016.v39n2/101-105" https://www.scielosp. org/pdf/rpsp/2016.v39n2/101-105.

9. Fontes GJF, Silva TG, Sousa JCM, Feitosa ANA, Silva ML, Bezerra ALD. Epidemiological profile of tuberculosis in Brazil from 2012 to 2016. Rev. Bra. Edu [Intemet]. Saúde. 2019 [cited 2020 nov 23]; 9(1):19-26. Avalaible from: http://dx.doi.org/10.17058/reci.v9i1.12249. 10. Santos DT, Garcia MC, Costa AANF, Pieri FM, Mier DAP, Albanese SPR et al. Latent tuberculosis infection in persons with HIVIAIDS, associated factors, and progression to active disease in a city in southern Brazil. Cad. Saúde Pública [Internet]. 2017 [cited nov 23]; 33(8):e00050916. Avalaible from: https://doi.org/10.1590/ 0102-311x00050916.

11. Rodrigues MW, Melo AGNC. Tuberculosis and schooling: A literature review. Revista Internacional de Apoyo a la Inclusión, Logopedia, Sociedad y Multiculturalidad [Internet]. 2018 [cited 2020 oct 17]; 4(2). Avalaible from: https://revistaselectronicas. ujaen.es/index.php/riai/article/view/4314.

12. Pereira AGL, Escosteguy CC, Valencia LIO, Magalhões MAFM, Medronho RA. Spatial distribution and socioeconomic context of tuberculosis in Rio de Janeiro, Brazil. Rev. Saúde Pública [Internet]. 2015 [cited 2020 oct 17]; 49:48. Avalaible from: http:// dx.doi.org/10.1590/50034-8910.2015049005470.

13. Gaspar RS, Nunes N, Nunes M, Rodrigues VP. Temporal analysis of reported cases of tuberculosis and of tuberculosis-HIV co-infection in Brazil between 2002 and 2012. J Bras Pneumol [Internet]. 2016 [cited 2020 oct 17]; 42(6):416-422. Avalaible from: https://doi.org/10.1590/s1806-37562016000000054

14. Lima SS, Vallinoto ACR, Machado LFA, Ishak MOG, Ishak R. Análise espacial da tuberculose em Belém, estado do Pará, Brasil. Rev Pan-Amaz Saude [Interne]. 2017 [cited 2020 oct 17]; 8(2):57-65. Avalaible from: http://dx.doi.org/10.5123/s217662232017000200007.

15. Cecilio HPM, Santos AL, Marcon SS, Latorre MRDO, Mathias TAF, Rossi RM. Tuberculosis mortality trend in the state of Paraná, Brazil - 1998-2012. Ciên. Saúde Colet [Internet]. 2018 [cited 2020 oct 17]; 23(1):241-248. Avalaible from: https://doi. org/10.1590/1413-81232018231.25242015.

16. Oliveira GS, Lima GRCC, Silva JX, Naves JOS, Monteiro Filho CMR. Laboratory identification of mycobacteria from respitatory samples of patients with suspected pulmonar tuberculosis in samples isolated at tha central laboratory of public health of the federal district (LACEN-DF). J. Health biol sci [Internet]. 2016 [cited 2020 oct 17]; 4(3):187-192. Avalaible from: http://dx.doi.org/10.12662/2317-3076jhbs. v4i3.712.p187-192.2016.

17. Ossalé Abacka KB, Koné A, Akoli Ekoya O, Bopaka RG, Lankoandé Siri H, Horo K. Tuberculose extrapulmonaire versus tuberculose pulmonaire: aspects épidémiologiques, diagnostiques et évolutifs. Rev Pneumol Clin [Internet]. 2018 [cited 2020 oct 17]; 72(6): 452-472. Avalaible from: https://doi.org/10.1016/j.pneumo.2018.09.008 18. Ferreira ALS, Nogueira LMV, Sá AMM, Ozela CS. Costs of the search for tuberculosis diagnosis: impact on the family economy. Rev Cuid [Interne]. 2018 [cited 2020 oct 17]; 9(3): 2400-12. Avalaible from: http://dx.doi.org/10.15649/cuidarte.v9i3.536. 19. De Oliveira LB, Costar CRB, Queiroz AAFLN, Araújo TME, Sousa KAA, Reis RK. Epidemiological analysis of tuberculosis/HIV coinfection. Cogitare Enfermagem [Internet]. 2018 [cited 2020 oct 17]; 23(1). Avalaible from: http://dx.doi.org/10.5380/ ce.v23i1.51016.

20. Scott L, da Silva P, Boehme CC, Stevens W, Gilpin CM. Diagnosis of opportunistic infections: HIV co-infections: Tuberculosis. Curr Opin HIV AIDS [Internet]. 2017 [cited 2020 nov 23]; 12 (2): 129-138.Avalaible from: https://dx.doi.org/10.1097\%2FCOH.0000000000000345.

21. Lima IB, Nogueira LMV, Guimarães RJPSE, Rodrigues ILA, André SR, Abreu PD et al. Spatial patterns of multidrug-resistant tuberculosis: correlation with sociodemographic variables and type of notification. Rev. Bras. Enferm [Internet]. 2020; [cited 2020 nov 23] 73(Suppl 5): e20190845. Avalaible from: http://dx.doi.org/10.1590/00347167-2019-0845

22. Queiroz AAR, Berra TZ, Garcia MCC, Popolin MP, Belchior AS, Yamamura M et al. Spatial pattern and temporal trend of mortality due to tuberculosis. Rev. Latino-Am. Enfermagem [Internet]. 2018 [cited 2020 nov 23]; 26: e2992. Avalaible from: https:// doi.org/10.1590/1518-8345.2049.2992.

23. Mathema B, Andrews JR, Cohen T, Borgdorff MW, Behr M, Glynn JR et al. Drivers of Tuberculosis Transmission. J Infect Dis [Internet]. 2017 [cited 2020 dec 12]; 216 (suppl 6): 644-S653. Avalaible from: https://doi.org/10.1093/infdis/jix354.

24. Wang $X$, Yin S, Li Y, Wang W, Du M, Guo W et al. Spatiotemporal epidemiology of, and factors associated with, the tuberculosis prevalence in northern China, 2010-2014, 2010-2014. BMC Infect Dis [Internet]. 2019 [cited 2020 dec 12]; 19(365). Avalaible from: https://doi.org/10.1186/s12879-019-3910-x. 